\title{
Solitary Fibrous Tumor Low Rectum Simulating Cancer
}

\author{
Rajaonarivony Tianarivelo, Mosa Fasoa, Andrianarijon Heritiana Nandrianina*, \\ Rakotomena Solonirina Davidà, Rahantasoa Finaritra Casimir Fleur Prudence, \\ Rakoto Ratsimba Hery Nirina
}

Unit of Digestive Surgery, Hospital Joseph Ravoahangy Andrianavalona, Antananarivo, Madagascar

Email address:

ahnandrianina@gmail.com (A. H. Nandrianina)

${ }^{*}$ Corresponding author

\section{To cite this article:}

Rajaonarivony Tianarivelo, Mosa Fasoa, Andrianarijon Heritiana Nandrianina, Rakotomena Solonirina Davidà, Rahantasoa Finaritra Casimir Fleur Prudence, Rakoto Ratsimba Hery Nirina. Solitary Fibrous Tumor Low Rectum Simulating Cancer. International Journal of Clinical Oncology and Cancer Research. Vol. 6, No. 3, 2021, pp. 115-117. doi: 10.11648/j.ijcocr.20210603.12

Received: March 29, 2021; Accepted: April 21, 2021; Published: July 13, 2021

\begin{abstract}
Introduction: The solitary fibrous tumor is a benign mesenchymal tumor of rare extra-pleural location. We report a case of solitary fibrous tumor observed in the lower rectum simulating cancer. The objective was to discuss the diagnostic and therapeutic management of the solitary fibrous in Madagascar. Observation: This is a sixty-five-year-old man, seen in consultation for dyskinesia, with no family history of neoplasia. The digital rectal examination revealed a large, non-budding, firm mass at the level of the right posterolateral surface with a lower pole located $2 \mathrm{~cm}$ from the anal margin, the remains of the physical examination are normal. Abdominal computed tomography showed a mass measuring $8 \times 7 \times 5.5 \mathrm{~cm}$ at the expense of the rectal wall of regular tissue density, without a mesenteric node or secondary localization. the biopsy had not found any malignant cells. This result is due to insufficiently deep biopsy samples of the mass, which led us to perform a complete surgical excision by coloprotectomy. The operative follow-up to which was simple. Immunohistochemical study of the surgical specimen confirmed the diagnosis with a positive CD 34 marker. The outcome was favourable without metastasis or recurrence after a six-month follow-up. Conclusion: The rectal localization of the solitary fibrous tumor is exceptional. The diagnosis is histological confirmed by the immunohistochemical study with a positive CD 34 marker.
\end{abstract}

Keywords: Excision, Immunohistochemistry, Mesenchymal Tumor, Rectum, Solitary Fibrous Tumor

\section{Introduction}

Solitary fibrous tumor is a benign mesenchymal tumor [1], which develops most of the time in the pleura. External pleural localization is exeptional, but it has recently been reported in various organs, particularly in the kidney, prostate and liver [2]. We report the case of a solitary fibrous tumor developed at the expense of the rectal wall at the Joseph Ravoahangy Andrianavalona University Hospital of Antananarivo. It was diagnosed with difficulty after surgical resection of the rectum due to the not specific sign. The objective was to discuss the diagnostic and therapeutic management in relation to literature.

\section{Observation}

A 65-year-old man, smoking, consulted for dyskinesia with progressive rectal syndrome for a year. The patient was in good general condition and showed no signs of anaemia or rectal bleeding. Abdominal palpation had not found any tumor. The digital rectal examination revealed a large, nonbudding, firm mass at the level of the right posterolateral surface with a lower pole located $2 \mathrm{~cm}$ from the anal margin. The recto sigmoidoscopy had found a slightly inflammatory rectal mucosa with a bulging of the posterior surface of the rectum partially narrowing the colonic lumen to $7 \mathrm{~cm}$ from the anal margin. Histologic examination of the biopsy specimens found a chronic non-specific proctitis. Abdominal computed tomography showed a mass measuring $8 \times 7 \times 5.5$ $\mathrm{cm}$ at the expense of the rectal wall of regular tissue density, hypervascularized in the arterial phase with more or less homogeneous enhancement after contrast injection (figure 1) 
with some lymphadenopathy in the arterial phase. Mesoreatum level, without urinary bladder and prostate invasion, no liver or pulmonary metastasis was observed. The CA 19-9 marker was discreetly high at $17.1 \mathrm{U} / \mathrm{ml}$. The patient had undergone coloprotectomy followed by coloanal anastomosis and protective ileostomy by open midline laparotomy. There was no incidence during the postoperative period. The histological results of the surgical part had macroscopically revealed a rectal mass, nodular, whitish, firm, well-defined, measuring $8 \times 7 \times 5.5 \mathrm{~cm}$ with nine nodes of $0.4 \times 1 \mathrm{~cm}$ long axis at the level of the mesorectum. Microscopically, a tumor proliferation made up of spindleshaped cells, grouped in long bundles in places, intersecting at right angles without cytonuclear atypia, nor mitosis had been objectified. The immunohistochemistry showed intense and diffuse tumor cell positivity to $\mathrm{CD} 34$ and negativity to smooth muscular actin, DOG-1, PS100 and desmin. Ki67 was evaluated at $1 \%$ confirming a solitary fibrous tumor of the rectum. After six months of follow-up, the patient was in good general condition, without sphincter disorder or recurrence

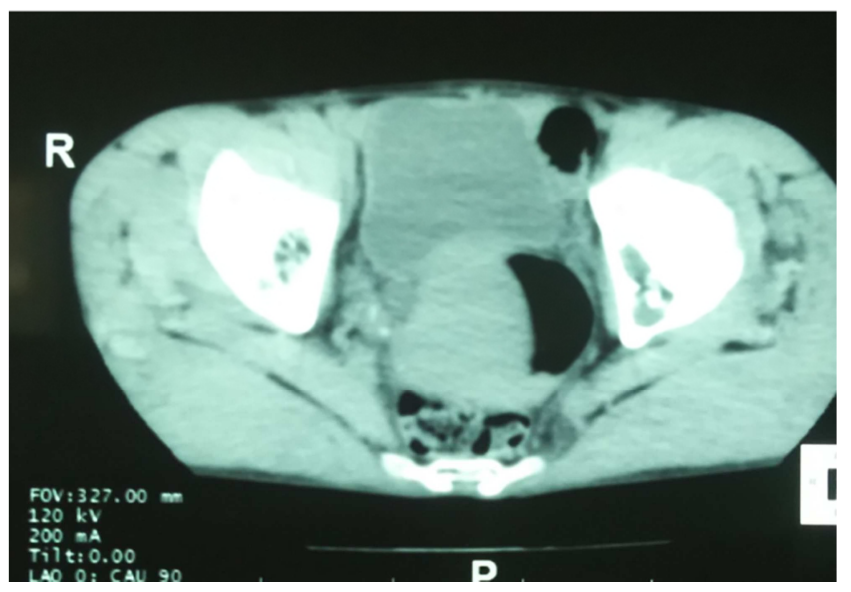

Source: Visceral surgery archive "A" at CHUJRA

Figure 1. Solitary fibrous tumor in the rectum.

\section{Discussion}

In the literature, solitary fibrous tumor is a exceptional benign tumor with a reported incidence of 2.8 cases per 100,000 people [3]. In our case, this is the first case of the rectal localisation of the solitary fibrous tumor in our country. Initially, it was described by Klemperer and Rabin in 1931 in the pleural, as localised fibrous mesothelioma [4]. Since its first description, a controversy persisted for many years on the origin of these tumors: mesothelial or mesenchymal submesothelial. It was only in the years 1980-1990 by the contribution of the study immunohistochemistry that the hypothesis of a mesenchymal origin was confirmed [1]. In the literature, the preferential localisation of a solitary fibrous tumor remains at the level of the pleural. The extra pleural locations reported are very varied: mesorectum [2], kidney [5], liver, retro-peritoneal, bladder, cervical, mammary [6]. We reported rectal localisation in our study. This pathology can be found at any age, with a median age 55 years old (50 to 70 years old) [7]. Our case is similar to the one in the literature; it was a 65-year-old man. In the literature, most solitary fibrous tumors were asymptomatic and discovered incidentally. The clinical manifestation depends mainly on the topography of the tumor. In $5 \%$ of cases, the tumors were discovered during hypoglycaemia [7] due to the secretion of insulin-like growth factor. It can be product by the tumor, but most lesions do not product this factor and only show signs of compression of adjacent organs [8]. In our case, it was manifested by dyskinesia with rectal syndrom due to the location in the lower rectum. As with any anorectal pathology. The rectal examination is essentially describing the characteristics of the tumor and already allowing to eliminate a malignant tumor. Macroscopically, it is a solid tumor, with a smooth area, often large, well circumscribed, which does not infiltrate the healthy parenchyma. Rectosigmoidoscopy with systematic biopsy should guide the diagnosis and rule out other anorectal pathologies. But superficial biopsy samples expose to low sensitivity and can induce a diagnostic error as in this case. As literature said, fine-needle aspiration biopsies rarely provide enough tissue for a definitive diagnosis due to the heterogeneous distribution of cell density within the tumor [8]. Histologically, it most often exists a great nonspecific architectural variability. The cells are spindle-shaped, are characterised by their irregular, nonnucleolus nucleus, in interlaced bundles arranging in an anarchic fashion within a fibrocollagenic stroma that is focally hyalinized. Their cytoplasm is eosinophilic [9]. This result had been confirmed in our case, but given the missing of a sign not specific to histology. Only the immunohistochemical results confirm the diagnosis of a solitary fibrous tumor, showing a positive staining among CD 34 and bcl-2 [3]. CD 34 labelling is strongly positive, showing diffuse cytoplasmic reactivity in 75 to $100 \%$ of cases, which makes it one of the most sensitive markers [10]. On Magnetic Resonance Imaging, the solitary fibrous tumor typically appears in hyposignal in $\mathrm{T} 1$ and $\mathrm{T} 2$. A T2 hypersignal is present in the event of necrosis, myxoid degeneration and hypercellular plaque. After the injection of gadolinium, the enhancement is often heterogeneous and intense [11]. As in our case, the computed tomography shows a formation of tissue density, well limited and intensely enhanced after the injection of contrast products due to a large vascular contingent [11] and our case did not receive imaging by magnetic resonance. In the literature, the differential diagnosis was difficult but mainly. It is necessary to rule out gastrointestinal stromal tumor [12], synovial sarcoma, reactive nodular fibrous pseudotumor of the gastrointestinal tract and mesentery and adenocarcinoma [13]. In the literature, the standard treatment for a solitary fibrous tumor is surgical excision, which is the only curative treatment [14].

In our case, we performed coloprotectomy followed by coloanal anastomosis and protective ileostomy by open midline laparotomy.

In the literature, a size of more than $10 \mathrm{~cm}$ and incomplete 
resection also correlates positively with local and metastatic recurrence [15] to prevent recurrence or distant metastasis even in the missing of any sign of malignancy. Solitary extra thoracic fibrous tumors are slow-growing tumors like benign mesenchymal tumors, although malignant various with aggressive local behaviour and metastasis may occur [16]. It therefore requires prolonged, close and long-term monitoring [17]. In our case, no recurrence, or loco regional or distant metastasis was encountered over a six-month follow-up.

\section{Conclusion}

Solitary fibrous tumor is rarely localised in the rectum. The rectal examination with a rectoscopy orient the diagnosis and can already eliminate malignant tumors by the macroscopic characteristics of the tumor. Sufficiently deep biopsy samples of the usually large, firm, regular and wellcircumscribed mass avoid negativity of the pathological results. Only the immunohistochemical examination by a CD 34 and bcl-2 staining confirm the diagnosis. Complete resection of the tumor, even if it is benign, helps prevent tumor recurrence, hence prolonged monitoring.

\section{References}

[1] Ravis TWD, Brambilla E, Muller HHK, Harris CC. World Health Organisation Classification of Tumours: Pathology and Genetics of Tumours of the Lung, Pleura, thymus and Heart. Lyon: IARC Press. 2004: 142.

[2] Yi B, Bewtra C, Yussef K, Silva E. Giant pelvic fibrous pelvic tumor obstructing the intestinal and urinary tract: case report and review of the literature. Suis Surg. 2007; 73: 478-80.

[3] Nagase T, Adachi I, Yamada T, Murakami N, Morita K, YoshinoY. Solitary fibrous tumor in the pelvic cavity with hypoglycemia: a case report. Surg today. 2005 ; 35: 181-4.

[4] Klemperer P, Coleman BR. Primary neoplasms of the pleural: a report of five cases. Am J Ind Med. 1931; 22 (1): 1-31.

[5] Wang j, Arber DA, Frankel K, Weiss LM. Large solitary fibrous tumor of the kidney: Report of two cases and review of the literature. Am. j. Surg. Pathol. 2001; 25 (9): 1194-9.
[6] Falconieri G, Lamovec J, Mirra M, Pizzolitto S. Solitary fibrous tumor of the mammary gland: A potential pitfall in breast pathology. Ann. Diag. pathol, 2004; 8: 121-25.

[7] Gold JS, Antonescu CR, Hajdu C. Clinicopathologic correlates of solitary fibrous tumors. Cancer 2002; 94: 105768 .

[8] Hoffmann H, Giger OT, Bubendorf L, Lardinois D. Contralateral recurrenc of a malignant solitary fibrous tumor of the pleura. Interact CardioVascThorac Surg 2011; 12: 306-7.

[9] Moran CA, Suster S, Koss MN. The spectrum of histologic growth patterns in benign and malignant fibrous tumor of the pleura. Sem. Diagnos. Pathol., 1992, 9, 169-180.

[10] Chan JKC. Solitary fibrous tumor: everywhere, and a diagnosis in vogue. Histopathology 1997; 31: 568-76.

[11] Cannard L, Debelle L, Laurent V, Béot S, Leclerc JC, Régent D. Imaging of solitary fibrous tumors of the pleural. Radiology Leaflets 2001; 41: 317-24.

[12] Miettinen M, Monihan JM, Sarlomo-RM, Kovatich AJ, Carr NJ, Emory TS, Sobin LH: Primary Gastrointestinal Stromal Tumors / Smooth Musclar Tumors (GISTs) in Omentum and Mesentery: Clinico-pathological and Immunohistochemical Study of 26 Cases, Am J Surg Pathol, 1999, 23 (9): 1109-18.

[13] Ryu HS, Heo I, Koh JS, Jin SH, Kang HJ, Cho SY: Primary monophasic synovial sarcoma occurring in the mesentery: case report of an extremely rare mesenteric sarcoma confirmed by molecular detection of a fusion transcript SYTSSX2, Korean J Pathol, 2012, 46 (2): 187-91.

[14] Vallat DAV, Dry SM, Fletcher CDM Atypical and solitary malignant fibrous tumors in external-thoracic locations: evidence of their comparability to inside thoracic tumors., Am J Surg Pathol, 1998, 22 (12): 1501-11.

[15] Daigeler A, Lehnhardt M, Langer S, Steinstraesser L, Steinau HU, Mentzel T, Kuhnen C: Clinopathologic findings in a case series of solitary external-thoracic fibrous tumors of soft tissue BMC Surg, 2006.

[16] Hiroaki S, Osamu K, Hiroshi Y, Matsuo N, Nobuhiro T, Atsushi I, Akihiro C, Hisashi G, Akinari M, Satoko I, Akinobu AC: solitary fibrous tumor giant inside-pelvic after Mesorectal J Gastroenterol 2010 3: 136-39.

[17] Cardillo G, Carbone L, Carleo F: Solitary fibrous tumors of the pleura: an analysis of 110 patients treated in a single institution: Ann Thorac Surg 2009 88: 1632-37. 